\title{
Digital Holography: \\ Evolution from a Research Topic to a Versatile Tool for the Inline $100 \%$ 3D Quality Control in Industry
}

\author{
Markus Fratz ${ }^{1}$, Tobias Beckmann ${ }^{1}$, Annelie Schiller ${ }^{1}$, Tobias Seyler ${ }^{1}$, Alexander Bertz ${ }^{1}$, Daniel Carl ${ }^{1}$, \\ Karsten Buse ${ }^{1,2}$ \\ ${ }^{1}$ Fraunhofer Institute for Physical Measurement Techniques IPM, Heidenhofstraße 8, \\ 79110 Freiburg, Germany, \\ ${ }^{2}$ Department of Microsystems Engineering, University of Freiburg, Georges-Köhler-Allee 102, \\ 79110 Freiburg, Germany \\ karsten.buse@ipm.fraunhofer.de
}

\begin{abstract}
:
Digital multiwavelength holography is a technique for precise 3D height measurements of optically rough surfaces. We demonstrate measurements on a milled surface, using four wavelengths between 514 and $532 \mathrm{~nm}$, and achieve precision in the submicrometer range. Height structures of $<400 \mathrm{~nm}$ can be resolved up to an unambiguous height of $370 \mu \mathrm{m}$ and with a lateral resolution of $7 \mu \mathrm{m}$. Acquisition times of $<400 \mathrm{~ms}$, including the time needed for parallel processing of the data, make our sensor a versatile tool for high-throughput $100 \%$ inspection in manufacturing environments.
\end{abstract}

Key words: Digital Holography, Inline Inspection, Phase Retrieval, Parallel Computing, GPU.

\section{Introduction}

Holography is used to precisely measure 3D structures of surfaces. Unlike photography, holography records both, the amplitude and the phase information or rather the height information. The use of electronic sensors for recording holograms dates back to the 1960s [1], and became more important in the 1990s [2],[3]. Computation times of one hologram larger than several seconds made holographic measurements suited for laboratory use but incompatible for controlling high throughput industrial processes [1],[3]. With the increasing computing power as well as the use of graphics processing units (GPU), the requirements of fast production cycles are met with computing times in the millisecond range [4],[5],[6],[7]. Furthermore, the steady increase in resolution and size of the camera chips makes holography nowadays a versatile tool for industrial applications.

Many technical surfaces are optically rough, i.e. the surface roughness exceeds the visible wavelength range. At the same time, these functional surfaces can have tolerances of only $10 \mu \mathrm{m}$, requiring measurement techniques with submicron precision. Moreover, structure heights of several hundred micrometers have to be measured unambiguously. Using multiwavelength holography these challenges can be met by recording interferograms at multiple wavelengths [8]. Out of these, interferograms at different synthetic wavelengths are computed. The synthetic wavelengths correspond to the beat frequencies. The wavelength difference determines the unambiguous height measurement range: The smaller the difference, the larger the synthetic wavelength. Large measurement ranges result in less accurate measurements.

The combination of more than two wavelengths allows cascading the measurement ranges, and thus increases the ratio of measurement range and accuracy.

Here we present new results of a digital multiwavelength holography sensor using four different wavelengths resulting in an accuracy of a few $100 \mathrm{~nm}$ covering an unambiguous height of $370 \mu \mathrm{m}$, generating $3072 \times 3072$ measurements on an area of $20 \times 20 \mathrm{~mm}^{2}$ in less than $400 \mathrm{~ms}$.

\section{Sensor description}

Four lasers emitting wavelengths between 514 and $532 \mathrm{~nm}$ are used, resulting in synthetic wavelengths between $752 \mu \mathrm{m}$ and $15 \mu \mathrm{m}$. The laser beams are coupled into fibers and connected to a fiber switch, which turns the 
individual laser beams on and off. The simplified beam path inside the sensor head is depicted in Fig. 1. After leaving the optical fiber (1), object and reference beams are generated by a beam splitter (2), with both beams being expanded to fit the size of the object and the camera chip. The reference beam is deflected by a mirror (3) mounted on a piezoelectric actuator. Thus the phase of the reference beam is shifted by moving the mirror slightly. The object beam illuminates the sample under test (4). An object lens (5) images the light scattered by the sample onto the camera sensor (6). The object and the reference beam are superimposed on the camera chip. The resolution of $3072 \mathrm{px} \times 3072 \mathrm{px}$ corresponds to a lateral resolution of $6.7 \mu \mathrm{m} \times 6.7 \mu \mathrm{m}$, at a measurement area of $20 \mathrm{~mm} \times 20 \mathrm{~mm}$.

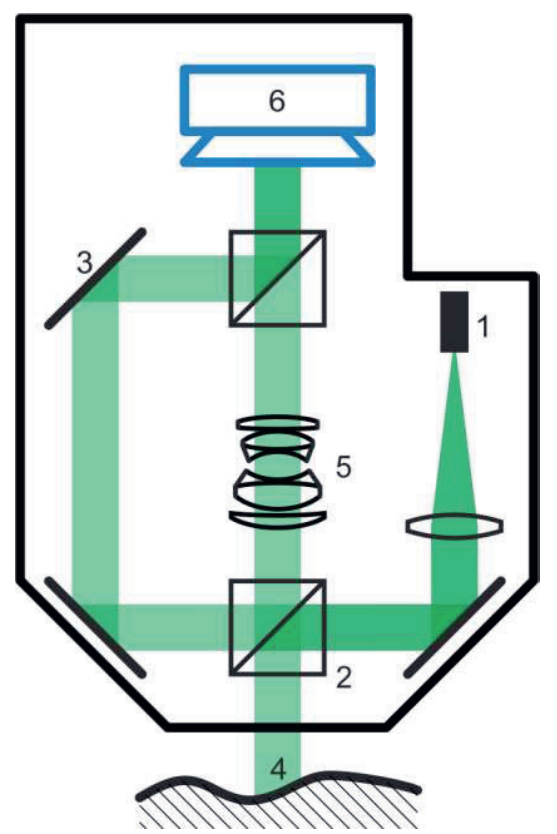

Fig. 1. Simplified sketch of the digital multiwavelength holography sensor: Light coming from the fiber (1) is split by the beam splitter (2) into object and reference beam. The mirror (3) in the reference beam is mounted on a piezoelectric actuator, used to introduce a phase shift to the reference beam. The object beam illuminates the object (4). The scattered light is imaged by an object lens (5) onto the camera (6), where it is superimposed with the reference beam.

Three phase-shifted holograms are recorded for each of the four different wavelengths, so all in all 12 images, each with $9 \mathrm{MPx}$ are taken. The phase shifts are determined by the method of Cai et al. [9].

To achieve fast production cycles, a CoaXPress camera is used for fast data acquisition and all phase-extraction computations, and filtering operations are performed in parallel using CUDA-programming on a consumer graphics card.

\section{Experimental results}

To demonstrate the unambiguous measurement range, the height topography of a 10 Euro Cent coin measured with the sensor described above is shown in Fig. 2. Despite the optically rough surface, height differences of $200 \mu \mathrm{m}$ can be resolved easily.

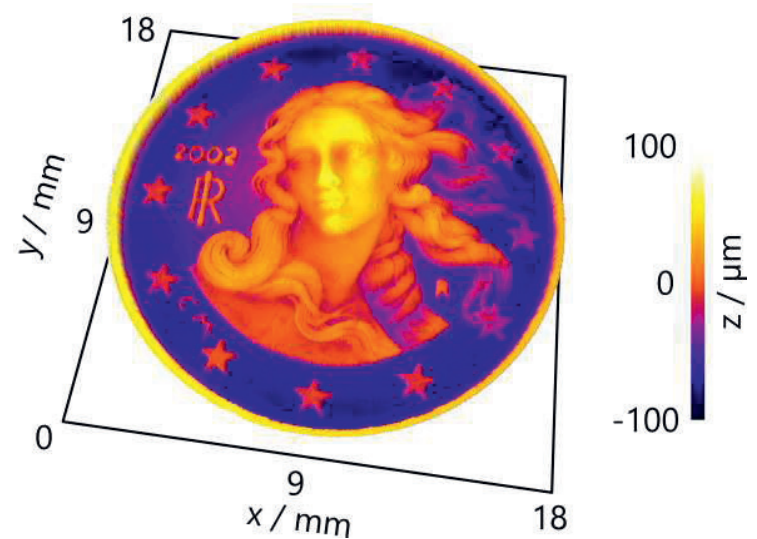

Fig. 2. Topography of a 10 Cent $€$ coin measured with the digital multiwavelength sensor.

To demonstrate the high axial resolution, the measurement results of a milled surface (see Fig. 3) with a borehole are shown in Fig. 4. The height map clearly resolves the milling grooves. The difference to a line fit on the height data along the blue line is shown as well in Fig. 4. As the surface structure can be seen in the height map, this is a combination of the sensor noise level and the surface roughness. Consequently, the sensor accuracy can be assumed to be better than $400 \mathrm{~nm}$. This measurement was taken in $85 \mathrm{~ms}$, and subsequent processing took just $300 \mathrm{~ms}$.

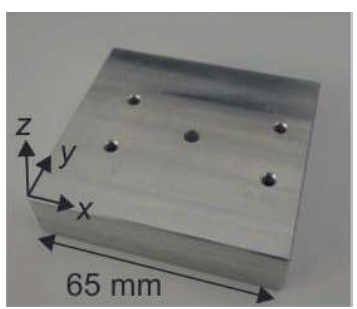

Fig. 3. Milled surface with boreholes, the metal piece has an area of $65 \mathrm{~mm} \times 60 \mathrm{~mm}$. 

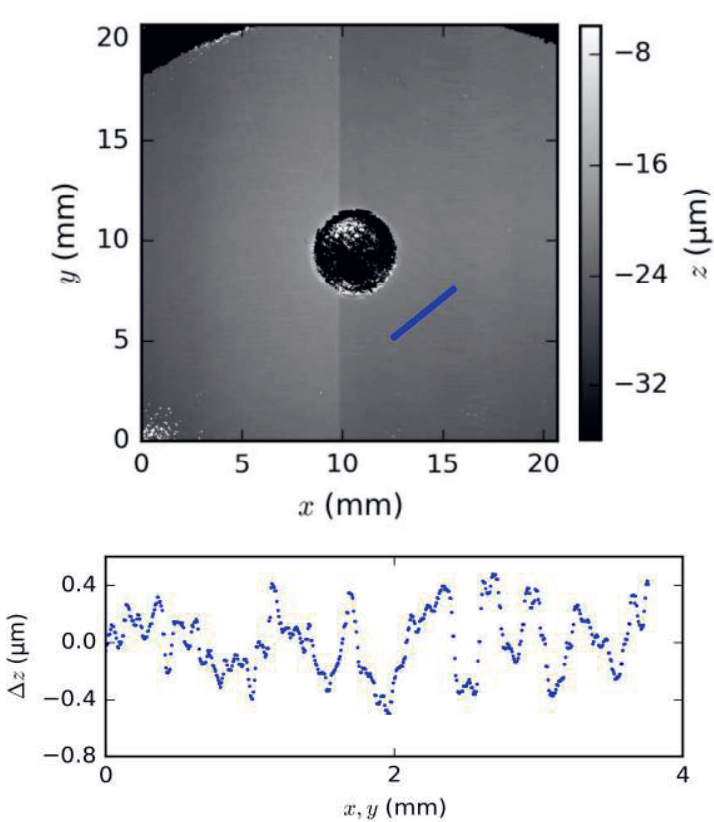

Fig. 4. Top: Height map of the milled surface with borehole. Bottom: The difference to a line fit in the section along the blue line displayed in the top part of the figure is shown.

\section{Inline Application}

The sensor head has an overall dimension of $440 \mathrm{~mm} \times 250 \mathrm{~mm} \times 105 \mathrm{~mm}$ and weights $11.5 \mathrm{~kg}$. In addition, a computer and a laser rack are needed. The working distance is typically $15 \mathrm{~mm}$. Figure 5 shows the sensor installed in a production line. There, it is used for $100 \%$ quality control of precision turned parts. Using three lasers emitting light from $633-636 \mathrm{~nm}$, an axial repeatability of $<1 \mu \mathrm{m}$ on an unambiguous measurement range of $500 \mu \mathrm{m}$ is achieved. A coin-sized measurement area of $18 \mathrm{~mm}$ in diameter corresponds to a lateral resolution of $6 \mu \mathrm{m} \times 6 \mu \mathrm{m}$. The time between two measurements is $1 \mathrm{~s}$, including image acquisition (<60 ms), data evaluation $(<150 \mathrm{~ms})$ and handling $(<800 \mathrm{~ms})$.

In 2015 a holographic sensor has been installed at Werner Giessler GmbH in Elzach, Germany. There the sensor is used for inline inspection of high pressure sealing surfaces for Diesel injection systems. $100 \%$ of the produced sealing parts are checked for correctness of various geometric features and micron-sized defects. Handling and inspection of each individual part is done within one second.

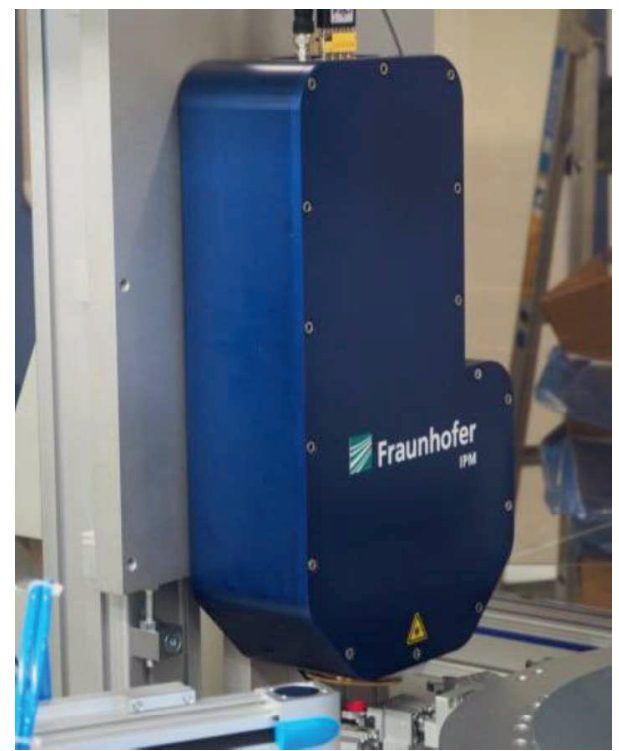

Fig. 5. Digital multiwavelength holography sensor installed at the company Werner Gießler $\mathrm{GmbH}$ in Elzach, inspecting one piece per second, synchronized with a production cycle of $1 \mathrm{~Hz}$.

\section{Perspectives}

The high precision of this technique shows the potential to be applied in optimizing milling and similar processes. Therefore, we are aiming at smaller sensor dimensions to be able to include this technique into machine tools for in-situ measurements providing feedback to the machine. Furthermore, measurements on moving objects at speeds of several $\mathrm{mm} / \mathrm{s}$ have been demonstrated recently [10], opening up new applications, simplifying the handling and thus increasing the upper limit for production cycles, as the measurement can be done continuously.

\section{Summary}

The high precision of digital multiwavelength holography was shown to be able to resolve structure heights of a few $100 \mathrm{~nm}$ with $370 \mu \mathrm{m}$ height unambiguity on an area of $20 \times 20 \mathrm{~mm}^{2}$ using parallel computing to generate $3072 \times 3072$ measurement points in less than $400 \mathrm{~ms}$.

Inline integration of a digital multiwavelength holography sensor has demonstrated the suitability of this technique for $100 \%$ quality control at a production cycle of $1 \mathrm{~Hz}$.

Miniaturized sensors for in-machine tools or holographic measurements on moving objects will push the field even further. 


\section{References}

[1] J. W. Goodman and R. W. Lawrence, Digital image formation from electronically detected holograms, Applied Physics Letters 11(3), 77-79 (1967); doi: 10.1063/1.1755043.

[2] U. Schnars and W. Jüptner, Direct recording of holograms by a CCD target and numerical reconstruction, Applied Optics 33(2), 179-181 (1994); doi: 10.1364/AO.33.000179.

[3] I. Yamaguchi, Phase-shifting digital holography, Optics Letters 22(16), 1268-1270 (1997); doi: 10.1364/OL.22.001268.

[4] D. Carl, M. Fratz, M. Pfeifer, D. M. Giel, and $H$. Höfler, Multiwavelength digital holography with autocalibration of phase shifts and artificial wavelengths, Applied Optics 48 (34), $\mathrm{H} 1-\mathrm{H} 8$ (2009); doi: 10.1364/AO.48.0000H1.

[5] M. Fratz and Daniel Carl, Novel industry ready sensors for shape measurement based on multi wavelength digital holography, Fringe 2013 (Springer 2014), pp. 479-484; doi: 10.1007/9783-642-36359-7_84.

[6] V. M. Bove, W. J. Plesniak, T. Quentmeyer, and J. Barabas, Real-time holographic video images with commodity PC hardware, Proceedings SPIE 5664, 255-262 (2005); doi: 10.1117/12.585888.

[7] N. Masuda, T. Ito, T. Tanaka, A. Shiraki, and T. Sugie, Computer generated holography using a graphics processing unit, Optics Express 14(2), 603-608 (2006); doi: 10.1364/OPEX.14.000603.

[8] C. Wagner, W. Osten, and S. Seebacher, Direct shape measurement by digital wavefront reconstruction and multiwavelength contouring, Optical Engineering 39(1),79-85 (2000); doi: 10.1117/1.602338.

[9] L. Z. Cai, Q. Liu, and X. L. Yang, Generalized phase-shifting interferometry with arbitrary unknown phase steps for diffraction objects, Optics Letters 29(2), 183-185 (2004); doi: 10.1364/OL.29.000183

[10] A. Schiller, T. Beckmann, M. Fratz, D. Belzer, A. Bertz, D. Carl, and K. Buse, Multiwavelength digital holography with spatial phase shifting on moving objects, in Imaging and Applied Optics 2016, DM3I.6 (2016); doi: 10.1364/DH.2016.DM3I.6. 